\title{
Optimisation de l'analyse multirésolution en ondelettes des signaux de choc. Application aux signaux engendrés par des roulements défectueux
}

\author{
Abderrazek Djebala ${ }^{1}$, Nouredine OuelaA ${ }^{1, a}$ et Nacer Hamzaoui ${ }^{2}$ \\ ${ }^{1}$ Groupe Comportement Dynamique des Structures et Maintenance Industrielle, Laboratoire de Mécanique et Structures, \\ Université de Guelma, BP 401, 24000 Guelma, Algérie \\ 2 Laboratoire Vibrations-Acoustique, INSA de Lyon, Bâtiment A. de St. Exupéry, 25bis avenue Jean Capelle, \\ 69621 Villeurbanne Cedex, France
}

Reçu le 28 décembre 2005, accepté le 19 juin 2007

\begin{abstract}
Résumé - L'objectif de cet article est de proposer l'analyse multirésolution en ondelettes en tant qu'outil efficace permettant d'améliorer la sensibilité des indicateurs scalaires (kurtosis et facteur de crête) pour l'identification des défauts mécaniques induisant des forces impulsives. En effet, ces indicateurs sont très sensibles aux variations dans le signal temporel dues aux chocs périodiques causés par le défaut. Néanmoins, leur fiabilité est immédiatement limitée en présence de niveaux intenses de bruit aléatoire associés à de faibles gravités du défaut. L'analyse Multirésolution en Ondelettes (AMRO) est proposée comme solution à ce problème. Adaptée pour un tel objectif, plusieurs de ses paramètres d'analyse sont choisis, voire optimisés. Initialement la méthode proposée est appliquée sur un signal simulé. Pour la validation expérimentale, plusieurs séries d'expériences ont été réalisées sur des roulements à billes et à rouleaux cylindriques, sur lesquels différents défauts ont été provoqués. Les mesures ont été prises dans différentes configurations, 210 signaux ont été mesurés dans plusieurs fréquences d'échantillonnage et vitesses de rotation sur un banc d'essais, l'application industrielle est faite sur un groupe turbo-alternateur.
\end{abstract}

Mots clés : Signaux de choc / analyse multirésolution en ondelettes / kurtosis / facteur de crête / détection des défauts

\begin{abstract}
Optimization of wavelet multiresolution analysis of shock signals. Application to the signals generated by defective rolling bearings. The aim of this paper is to propose the wavelet analysis as an effective tool allowing to improve the sensitivity of scalar indicators (Kurtosis and crest factor) for the identification of mechanical faults inducing impulsive forces. Indeed, these indicators are very sensitive to the variations in temporal signal due to the periodic shocks caused by the defect. Nevertheless, their reliability is immediately limited by the presence of high level of random noise associated to a small gravity of the defect. Wavelet Multiresolution Analysis (WMRA) is proposed as a solution to this problem. Adapted for such objective, several of its parameters were chosen, even optimized. Initially, the proposed method is applied to simulated signal. For the experimental validation, several series of experiments were realized on ball and cylindrical roller bearings, on which various defects were caused. Measurements were taken with various configurations, 210 signals were measured at different sampling rates and rotation speeds on a laboratory test rig, the industrial application is carried out on a turbo alternator.
\end{abstract}

Key words: Shocks signals / wavelet multiresolution analysis / kurtosis / crest factor / defects detection

\section{Introduction}

Plusieurs éléments de machines produisent des chocs lorsqu'ils sont partiellement ou totalement endommagés. La mise en place d'une maintenance conditionnelle basée

a Auteur correspondant : n_ouelaa@ yahoo.fr sur un suivi vibratoire rigoureux semble une solution appropriée pour la détection précoce de ces défauts, chose qui n'est malheureusement pas toujours évidente. En effet, dans les conditions normales la distribution des amplitudes est du type gaussien, si un défaut se manifeste une modification apparaît sur le signal sous forme d'impulsions périodiques. 


\section{Nomenclature}

\begin{tabular}{|llll|}
\hline$a$ & paramètre d'échelle & $F_{0}$ & fréquence de résonance de phase \\
$A_{i}$ & approximations & $K$ & kurtosis \\
$b$ & paramètre de translation & $M_{2}$ et $M_{4}$ & moments statistiques d'ordre 2 et 4 \\
$c A_{i}$ & coefficients d'approximation & $N$ & nombre d'échantillons \\
$c D_{i}$ & coefficients de détail & $n$ & nombre de niveaux \\
$F C$ & facteur de crête & $N_{\mathrm{r}}$ & vitesse de rotation \\
$C W T$ & Continuous Wavelets Transform & $Q$ & facteur de qualité \\
$D_{i}$ & détails & $s(t)$ & signal \\
$d b N$ & ondelettes de Daubechies d'ordre $N$ & $s^{\prime}(t)$ & signal reconstruit \\
$D W T$ & Discrete Wavelet Transform & $\bar{s}$ & valeur moyenne \\
$F_{\mathrm{c}}$ & fréquence de choc & $T_{\mathrm{d}}$ & pas de peigne de Dirac \\
$F_{\mathrm{L}}$ & fréquence d'oscillation libre & $t$ & temps \\
$F_{\mathrm{max}}$ & fréquence maximale & $\tau$ & temps de relaxation \\
$F_{\mathrm{e}}$ & fréquence d'échantillonnage & $\psi(t)$ & ondelette mère \\
\hline
\end{tabular}

Dans le cas d'une surveillance vibratoire dans le domaine temporel, des études ont montré le grand apport des indicateurs scalaires, tels que le kurtosis et le facteur de crête, pour l'identification des forces impulsives dues aux défauts $[1,2]$. Néanmoins, le kurtosis semble plus sensible que le facteur de crête, en particulier à la vitesse de rotation et à la bande de fréquence et trouve sa grande efficacité de détection aux bandes étroites aux hautes fréquences, surtout pour des défauts naissants. La fiabilité des indicateurs scalaires est aussi bien prouvée dans l'analyse statistique du bruit et des vibrations des roulements endommagés [3] où l'influence de la vitesse de rotation et du type du défaut a été évoquée.

À cause des effets de masque, dont les origines sont diverses, ces impacts sont souvent noyés dans des perturbations, bruit de fond et d'autres constituants de la machine viennent polluer le signal et rendent la détection difficile voire impossible. Les indicateurs scalaires étant limités, plusieurs méthodes de débruitage ont été proposées pour améliorer leur sensibilité, utilisant la soustraction spectrale $[4,5]$, le filtrage adaptif [6] et le débruitage mixte [7]. En général plusieurs autres méthodes existent, Donoho [8] est le premier à établir une méthode de débruitage basée sur la transformée en ondelettes. Dans [9], une méthode basée sur l'ondelette de Morlet a été testée avec succès sur les engrenages et les roulements, elle permet un débruitage bien meilleur que celui de Donoho.

Le développement de la distribution temps-fréquence a permis la mise en place de plusieurs techniques fiables telles que la transformée de Fourier à court terme, la distribution de Wigner-ville et la transformée en ondelettes. Cette dernière, offrant un compromis entre résolution temporelle et fréquentielle, est la plus récente. Plusieurs applications de la transformée en ondelettes pour la détection des défauts ont été proposées, utilisant la transformée en ondelettes continue enrichie avec des techniques récentes [10-15], discrète (ou récemment appelée Analyse Multirésolution) [16-19] et l'analyse par paquet d'ondelettes [20]. Une synthèse intéressante est très bien présentée dans [21].
La supériorité de l'analyse en ondelettes face à beaucoup d'autres méthodes, y compris celles tempsfréquence, a été prouvée dans plusieurs études. Dans [19] elle a permis la détection d'un défaut d'engrenage que la STFT n'a pas pu détecter ou encore dans [22] elle a permis la détection des défauts dans un moteur électrique. Face à trois autres méthodes basées sur l'analyse bispectrale [23], elle a encore une fois prouvé son efficacité par une détection claire des défauts de roulements dans un moteur à induction.

L'objectif de ce travail est de proposer une méthode basée sur l'optimisation de l'analyse multirésolution en ondelettes. Adaptée à la détection des défauts induisant des chocs, elle permet l'amélioration de la sensibilité des indicateurs scalaires tout en restant efficace aussi bien pour les basses fréquences que pour les plus hautes d'entre elles.

Dans la section 2 le kurtosis et le facteur de crête sont présentés et leur limite prouvée, l'influence du bruit est également étudiée. Un bref rappel sur l'analyse multirésolution en ondelettes comprenant une optimisation de quelques-uns de ses paramètres est présenté dans la section 3. Après une simulation théorique dans la section 4, la méthode proposée est appliquée sur des signaux expérimentaux de roulements neufs et endommagés mesurés avec différentes fréquences d'échantillonnage et à plusieurs vitesses de rotation dans la section 5. Une application industrielle est enfin entamée sur une machine de production dans la section 6 .

\section{Indicateurs scalaires de détection}

Les indicateurs scalaires associent à un signal vibratoire, observé le plus souvent sous sa forme temporelle sur une durée déterminée en relation avec la cinématique de l'installation, un nombre ou scalaire [2]. Divers indicateurs sont utilisés dans le suivi vibratoire des machines tournantes, on peut citer à cet effet; la valeur efficace, la valeur crête ou une combinaison de ces deux grandeurs représentée par le kurtosis et le facteur de crête. Le 
kurtosis est un paramètre statistique permettant d'analyser la répartition des amplitudes vibratoires contenues dans un signal temporel. Il correspond au moment d'ordre quatre normé [4]. Son expression est de la forme :

$$
K=\frac{M_{4}}{M_{2}^{2}}=\frac{\frac{1}{N} \sum_{i=1}^{N}(s(i)-\bar{s})^{4}}{\left[\frac{1}{N} \sum_{i=1}^{N}(s(i)-\bar{s})^{2}\right]^{2}}
$$

avec $M_{4}$ et $M_{2}$ respectivement moments statistiques d'ordre 4 et $2, N$ le nombre d'échantillons du signal et $\bar{s}$ sa valeur moyenne donnée par :

$$
\bar{s}=\frac{1}{N} \sum_{i=1}^{N} s(i)
$$

Le facteur de crête correspond au rapport entre la valeur crête d'un signal (en valeur absolue) et sa valeur efficace. Son expression est :

$$
F C=\frac{\sup |s(i)|}{\sqrt{\frac{1}{N} \sum_{i=1}^{N}[s(i)]^{2}}}
$$

Afin de vérifier l'aptitude du kurtosis et du facteur de crête à identifier les forces impulsives périodiques, une étude comparative avec deux autres indicateurs scalaires couramment utilisés a été entamée. Pour ce faire, quatre signaux typiques ont été modélisés. Le premier signal « S1 (Fig. 1a) est la somme de trois sinusoïdes, le deuxième « $\mathrm{S} 2 »$ (Fig. 1b) est une fonction aléatoire représentée par un niveau significatif de bruit blanc gaussien, quant au troisième « S3» (Fig. 1c) il est égal à la somme des signaux « S1 $»$ et « $\mathrm{S} 2 »$, enfin le quatrième « $\mathrm{S} 4$ » (Fig. 1d) représente un signal simulant des impacts périodiques à $200 \mathrm{~Hz}$ pouvant modéliser des chocs induits par un défaut de roulement.

Pour l'ensemble des signaux, quatre indicateurs scalaires ont été calculés; le kurtosis, le facteur de crête, le rms et l'énergie vibratoire. D'après la figure 2 , il apparaît clairement que le kurtosis est l'indicateur le plus sensible aux chocs, sa valeur importante du signal « S4 » ne met aucun doute sur ce fait. Le facteur de crête semble lui aussi sensible aux chocs mais bien loin du kurtosis. Enfin, le rms et l'énergie vibratoire affichent, au contraire, des valeurs importantes pour les trois autres signaux. En effet, ils sont sensibles aux amplitudes du signal et non pas au phénomène induisant les chocs, de ce fait il est inutile de les utiliser comme indicateurs de l'état de dégradation des roulements, par contre leur variation dans le temps peut avoir une signification sur l'aggravation du défaut [1].

Plusieurs études ont montré que le kurtosis d'un signal aléatoire sans défaut, dont la distribution des amplitudes est normale, est égal à trois. Le facteur de crête du même signal peut varier de 3 à 6 . De ce fait, ils sont indicateurs de la présence d'un choc si leurs valeurs dépassent ces seuils $[1,2]$.
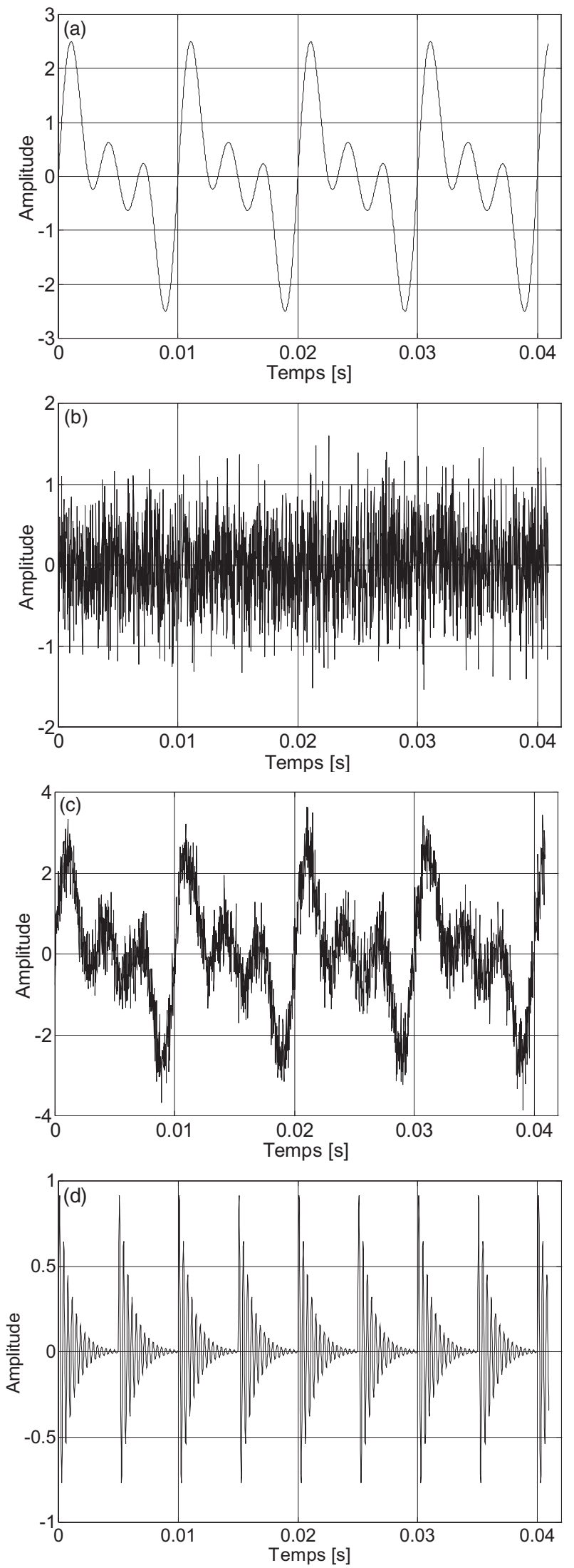

Fig. 1. Signaux simulant : (a) somme de trois sinusoïdes, (b) bruit blanc gaussien, (c) somme des signaux a et b, (d) impacts à $200 \mathrm{~Hz}$. 


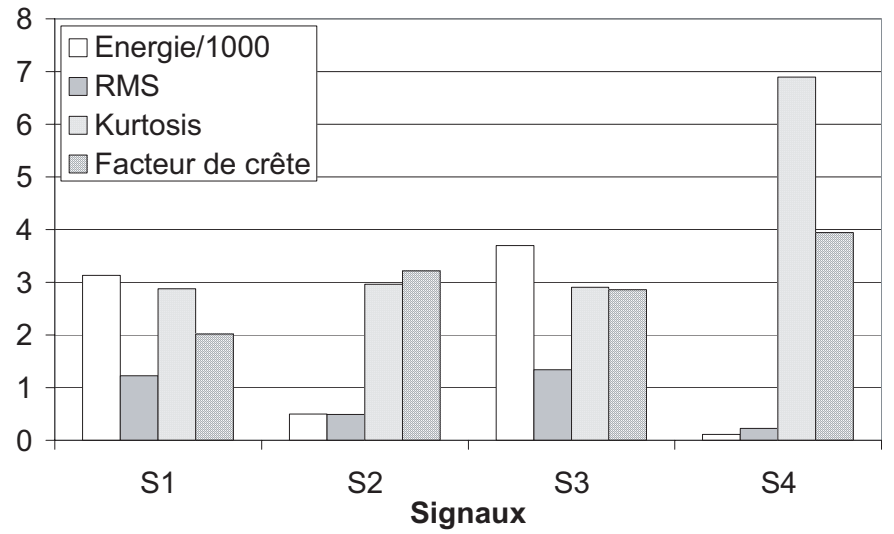

Fig. 2. Valeurs des indicateurs scalaires des quatre signaux.

\subsection{Modélisation des défauts induisant des forces impulsives}

Les impacts dus au défaut sont modélisés par le produit de convolution entre la réponse d'une résonance d'un système linéaire dissipatif à un degré de liberté avec amortissement de type visqueux, par un peigne de Dirac de période $T_{\mathrm{d}}$, correspondant à la fréquence de répétition des chocs [2]. Ceci se traduit mathématiquement par l'expression :

$$
s(t)=y(t)^{*} \sum_{k=0}^{\infty} \delta\left(t-k T_{\mathrm{d}}\right)
$$

avec

$$
y(t)=e^{-t / \tau} \sin 2 \pi F_{\mathrm{L}} t
$$

et

$$
\tau=\frac{Q}{\pi F_{0}}, \quad F_{\mathrm{L}}=F_{0} \sqrt{1-\frac{1}{4 Q^{2}}} \quad \text { et } \quad Q \succ 1 / 2
$$

où $\tau, F_{\mathrm{L}}, F_{0}$ et $Q$ représentent respectivement le temps de relaxation, la fréquence d'oscillation libre, la fréquence de résonance de phase et le facteur de qualité.

\subsection{Influence du bruit et des autres composantes de la machine sur la sensibilité des indicateurs scalaires}

La figure 3 montre le même signal de la figure 1d auquel on a ajouté un niveau significatif de bruit blanc gaussien et dix composantes pour simuler les basses fréquences $(20,23,24,26,29,44,45,55,60$ et $130 \mathrm{~Hz})$, de telle façon que le signal original représente $17 \%$ du signal bruité (rapport entre l'énergie du signal original sur celle du signal bruité). En effet, ces fréquences simulent les autres composantes de la machine qui n'ont pas le caractère aléatoire comme le bruit mais plutôt sinusoïdal telles que les fréquences de rotation et leurs harmoniques par exemple. Ces composantes ont autant d'influence, sur la sensibilité des indicateurs scalaires, que le bruit. Il semble par conséquent important de les prendre en considération.

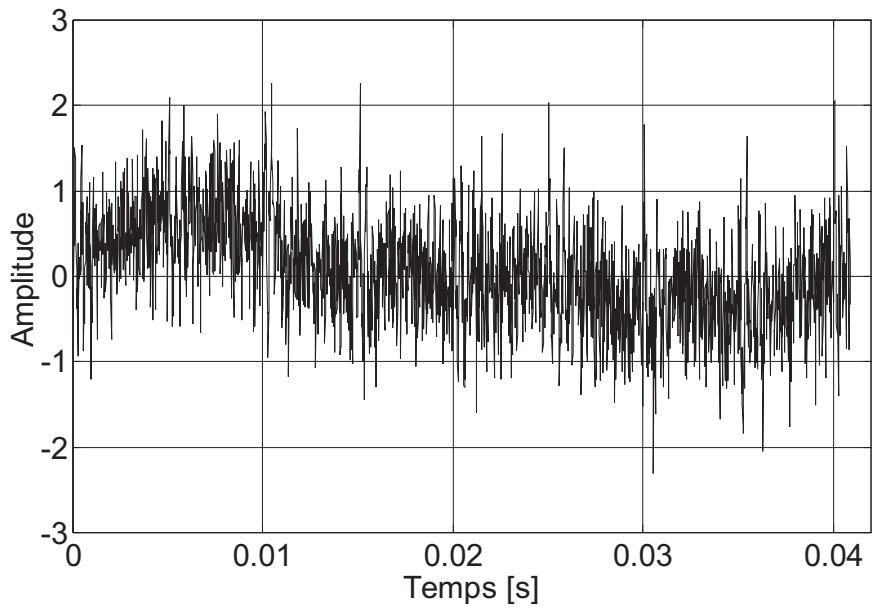

Fig. 3. Signal bruité; $F_{\mathrm{e}}=50000 \mathrm{~Hz}$.

Sur la figure 3 on constate que les impacts dus au défaut sont complètement masqués et l'information sur sa nature est ainsi perdue. Dans ce cas, les indicateurs scalaires diminuent sensiblement et atteignent des valeurs qui ne sont d'ailleurs pas significatives d'une présence d'un choc, à savoir 3,57 (soit un rapport de diminution de 1,1) pour le facteur de crête et 3,05 (soit un rapport de 2,26) pour le kurtosis, ce qui illustre sa grande sensibilité au bruit.

\section{Optimisation de l'analyse multirésolution en ondelettes des signaux de choc, méthode proposée}

\subsection{Théorie de l'AMRO}

La transformée en ondelettes est une transformation mathématique qui représente un signal $s(t)$ en terme de fonctions issues de la dilatation et de la translation d'une fonction singulière appelée ondelette mère $\psi(t)$. La famille d'ondelettes se met sous la forme :

$$
\psi_{a, b}(t)=\frac{1}{\sqrt{a}} \psi\left(\frac{t-b}{a}\right)
$$

avec $a$ paramètre d'échelle et $b$ paramètre de translation. En notant $\psi^{*}(t)$ la conjuguée de $\psi(t)$, la transformée continue en ondelette $(C W T)$ du signal $s(t)$ est définie par :

$$
C W T(a, b)=\frac{1}{\sqrt{a}} \int_{-\infty}^{+\infty} s(t) \psi^{*}\left(\frac{t-b}{a}\right) \mathrm{d} t
$$

La transformée en ondelettes discrète $(D W T)$ est une discrétisation de la transformée en ondelettes continue $(C W T)$. En remplaçant respectivement $a$ et $b$ par $2^{m}$ et $n 2^{m}$, avec $m$ et $n$ des entiers, son expression devient :

$$
D W T(m, n)=2^{\frac{-m}{2}} \int_{-\infty}^{+\infty} s(t) \psi^{*}\left(2^{-m} t-n\right) \mathrm{d} t
$$




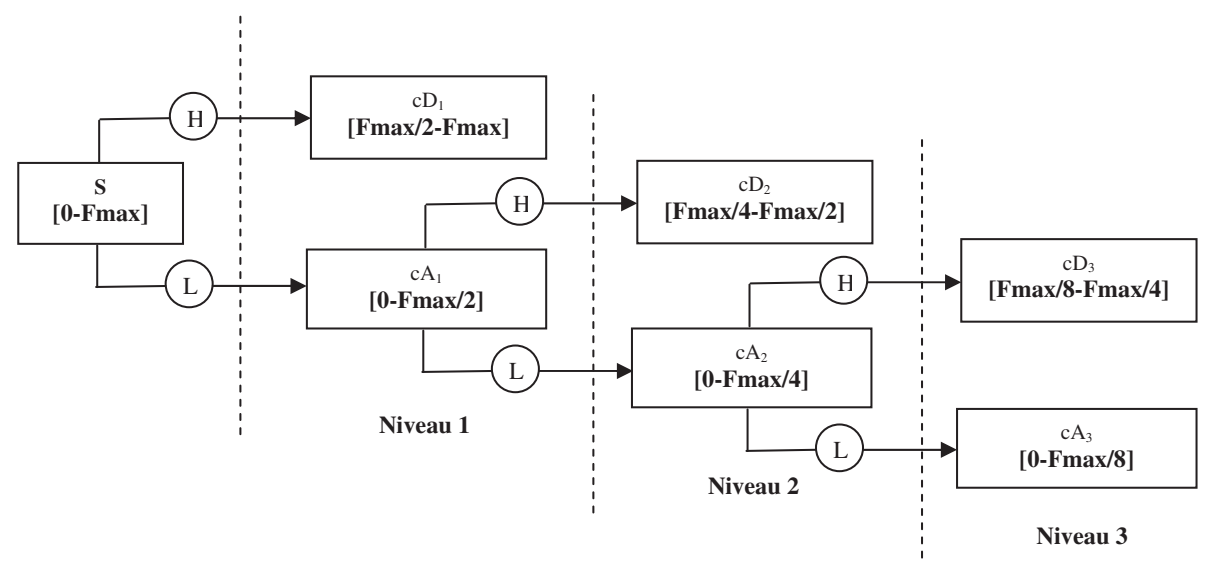

Fig. 4. Décomposition en cascade en trois niveaux.

Une version pratique de cette transformée, appelée analyse multirésolution en ondelettes (AMRO), a été introduite pour la première fois par Mallat en 1989, elle consiste à faire passer le signal $s(t)$ dans deux filtres passe-bas $(L)$ et passe-haut $(H)$. À ce niveau, deux vecteurs seront obtenus, $c A_{1}$ et $c D_{1}$. Les éléments du vecteur $c A_{1}$ sont appelés coefficients d'approximation, ils correspondent aux plus basses fréquences du signal, tandis que les éléments du vecteur $c D_{1}$ sont appelés coefficients de détail, ils correspondent aux plus hautes d'entre elles. La procédure peut être répétée avec les éléments du vecteur $c A_{1}$ et successivement avec chaque nouveau vecteur $c A_{j}$ obtenu. Le processus de décomposition peut être répété $n$ fois, avec $n$ le nombre maximal de niveaux. Lors de la décomposition, le signal $s(t)$ et les vecteurs $c A_{j}$ subissent un sous échantillonnage, c'est la raison pour laquelle les coefficients d'approximation $c A_{j}$ et de détail $c D_{j}$ passent à nouveaux à travers deux filtres de reconstruction $(L R)$ et $(H R)$. Deux vecteurs en résultent : $A_{j}$ appelés approximations et $D_{j}$ appelés détails, satisfaisant la relation :

$$
\begin{aligned}
& A_{j-1}=A_{j}+D_{j} \\
& s=A_{j}+\sum_{i \leqslant j} D_{i}
\end{aligned}
$$

où $i$ et $j$ sont des entiers.

Désignant par $F_{\max }$ la fréquence maximale du signal mesuré, la bande de fréquence de chaque niveau $(i)$ revient à $\left[0-\frac{F_{\max }}{2^{i}}\right]$ pour les approximations et $\left[\frac{F_{\max }}{2^{i}}-\frac{F_{\max }}{2^{i-1}}\right]$ pour les détails. La figure 4 représente un exemple de décomposition pour $n=3$.

\subsection{Approche proposée}

L'utilisation de la transformée en ondelettes discrète revient à passer le signal sous un microscope. Le filtrage en cascade permet de visualiser chaque partie du signal avec une résolution adaptée à son échelle. Plusieurs signaux de bandes fréquentielles différentes seront alors obtenus, nommés détails et approximations.
L'approche utilisée est de chercher les impacts, signe de la détérioration du roulement, parmi plusieurs autres composantes du signal ainsi que le bruit. Le kurtosis utilisé comme critère de recherche permet de déceler ces impacts en choisissant le détail ou l'approximation, issus de la décomposition en ondelettes du signal, qui permet la meilleure détection temporelle (kurtosis max) ou fréquentielle par le spectre d'enveloppe.

Bien évidemment, ne garder que l'information utile revient à perdre, volontairement d'ailleurs, toutes les autres. Dans ce cas, toute composante fréquentielle n'induisant pas des forces impulsives sera automatiquement écartée. De ce fait, un balourd ou un mésalignement du roulement ne peuvent être détectés.

En réalité cette approche a été utilisée par plusieurs auteurs [16-21] sans toutefois un choix rationnel des paramètres d'analyse. La contribution essentielle de ce travail est d'adapter la transformée en ondelettes discrète à l'analyse des signaux de choc et ceci par le choix et l'optimisation de plusieurs paramètres influents : type d'ondelettes, niveau optimal, nombre de niveaux, fréquence de choc ou vitesse de rotation et enfin la fréquence d'échantillonnage.

\subsubsection{Choix optimal du nombre des niveaux de l'AMRO}

Le principe de ce choix est de ne conserver que les niveaux qui comprennent l'information. La fréquence maximale de l'approximation du niveau final $F_{\max }\left(A_{n}\right)$ doit impérativement être égale à la fréquence du choc et au moins quelques unes de ses harmoniques afin de confirmer qu'il s'agit bien d'une fréquence d'un défaut. Pratiquement, on considère que trois sont suffisantes, ce qui donne :

$$
F_{\max }\left(A_{n}\right)=\frac{F_{\max }(s)}{2^{n}}=3 F_{\mathrm{c}}
$$

Donc, le nombre de niveaux doit à son tour satisfaire :

$$
n=1,44 \log \left(\frac{F_{\max }(s)}{3 F_{\mathrm{c}}}\right)
$$




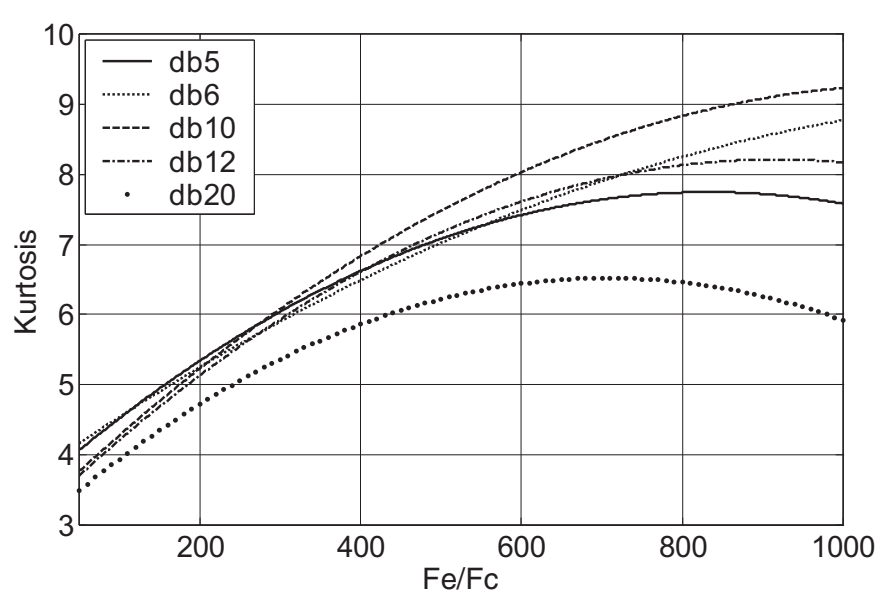

Fig. 5. Kurtosis du signal reconstruit en fonction du rapport $F_{\mathrm{e}} / F_{\mathrm{c}}$ pour différentes ondelettes de Daubechies.

\subsubsection{Choix du vecteur optimal de la décomposition}

L'AMRO permet d'avoir un certain nombre de vecteurs constitués de détails, correspondant aux hautes fréquences, et des approximations correspondant aux plus basses d'entre elles. Le vecteur optimal de la décomposition est, dans notre cas, celui qui permet la détection du défaut avec la meilleure résolution possible, cela nous conduit à sélectionner le mieux filtré. Pour ce faire, les indicateurs scalaires sont utilisés comme critères de sélection, le vecteur optimal sera donc celui ayant le kurtosis et le facteur de crête les plus importants.

$$
V_{\mathrm{opt}}=C A_{j} \quad \text { ou } \quad C D_{j}\left(K_{\max }, F C_{\max }\right)
$$

À partir des coefficients d'ondelettes de ce vecteur, un signal $s^{\prime}(t)$ sera reconstruit ayant un kurtosis et un facteur de crête plus significatifs que ceux du signal d'origine $s(t)$.

\subsubsection{Choix optimal d'une famille d'ondelettes et d'une fréquence d'échantillonnage du signal}

Il y a dans la littérature plusieurs familles d'ondelettes dont les qualités varient en fonction de plusieurs critères. Les ondelettes sont choisies surtout en fonction du type d'analyse voulu et de leur régularité. Après avoir éliminé les familles inadaptées pour l'algorithme rapide de l'AMRO (ondelettes de Morlet, ondelettes « chapeau mexicain » et ondelettes de Meyer), notre choix s'est porté sur les ondelettes de Daubechies $(d b N)$, qui semblent les mieux adaptées pour une telle analyse $[16,17,20]$, le problème reste dans le choix de l'ondelette elle-même.

Dans le cas présent, l'ondelette choisie sera celle qui permet un kurtosis maximal du signal reconstruit. Après une pré-sélection, les Daubechies 5, 6, 10, 12 et 20 ont été retenues, le kurtosis du signal reconstruit calculé pour différents rapports $F_{\mathrm{e}} / F_{\mathrm{c}}$ est représenté par la figure 5 .

On constate que la $d b 5$ est la mieux adaptée pour les petits rapports $F_{\mathrm{e}} / F_{\mathrm{c}}$ (jusqu'à 300 ), tandis que la $d b 10$ pour les grands (jusqu'à 1000) où le kurtosis atteint ses valeurs maximales ce qui peut correspondre pratiquement à des fréquences d'échantillonnage importantes associées à de faibles fréquences de choc (ou vitesses de rotation). En conclusion, en choisissant un rapport $F_{\mathrm{e}} / F_{\mathrm{c}}$ maximal (selon les capacités de l'appareillage de mesure) et son ondelette correspondante, on peut assurer un filtrage maximal du signal mesuré. En effet si la fréquence de choc est trop grande le temps de relaxation peut dépasser la période entre deux chocs successifs, les indicateurs scalaires perdent alors toute leur efficacité, or des études ont montré que pour profiter pleinement de leur pouvoir de détection, la répétition des chocs doit être supérieure de 2,5 à 3 fois le temps de relaxation pour le kurtosis et de 7 à 13 fois pour le facteur de crête $[1,2]$. La condition 12 permet de minimiser le nombre de niveaux et par suite le temps de calcul.

\section{Application sur un signal théorique}

La figure 6a représente le signal reconstruit après l'application de la méthode proposée sur le signal bruité de la figure $1 \mathrm{~d}$, les impacts dus aux chocs sont complètement visibles, quant à leur fréquence, elle est mise en évidence par le spectre d'enveloppe des coefficients d'ondelettes (Fig. 6b). Le signal reconstruit est extrait à partir du détail 4, sa bande fréquentielle est de $1562,5 \mathrm{~Hz}$ jusqu'à $3125 \mathrm{~Hz}$, la fréquence propre simulée $(2800 \mathrm{~Hz})$ est par conséquent incluse dans cet intervalle, ce qui revient à une bande passante autour de la fréquence de résonance. Dans ce cas les indicateurs scalaires augmentent considérablement, le kurtosis reste très sensible et atteint 5,85 contre 4,36 pour le facteur de crête.

\section{Application expérimentale sur des signaux de roulements défectueux}

Plusieurs expériences, ayant pour but de valider la méthode proposée, ont été réalisées sur des roulements à une rangée de billes du type 6200 montés sur un banc d'essai adéquat. Petits et grands défauts sont provoqués sur la bague extérieure, intérieure et sur la bille à l'aide d'un outil en diamant tournant à une très grande vitesse (50000 tr.min $\left.{ }^{-1}\right)$. D'autres défauts ont été provoqués sur la bague extérieure de roulements à rouleaux cylindriques montés sur un tour parallèle. Les défauts sont simulés sur toute la largeur du roulement, ils ont les dimensions suivantes:

- petit défaut $(L \times P)=(1 \times 0,3) \mathrm{mm}$;

- grand défaut $(L \times P)=(1,3 \times 0,7) \mathrm{mm}$.

avec $L$ la largeur du défaut et $P$ sa profondeur. La figure 7 montre l'exemple d'un petit défaut simulé sur la bague intérieure d'un roulement du type 6200. En réalité, vu la forme concave du chemin de roulement, la profondeur du défaut sous le point de contact de la bille est beaucoup plus faible que celle du cratère extérieur $\left(P^{\prime}\right)$ qui, dans le cas de la figure 7 , est égale à $0,8 \mathrm{~mm}$. Les mesures ont été réalisées par un analyseur B\&K 2035 muni 
Tableau 1. Détails concernant les expériences.

\begin{tabular}{|c|c|c|c|c|c|c|c|c|c|}
\hline \multirow{2}{*}{$\begin{array}{l}\text { Type du roulement } \\
\mathrm{N}^{\circ} \mathrm{du} \text { roulement } \\
\end{array}$} & \multicolumn{7}{|c|}{$\begin{array}{c}6200 \\
\text { (sur banc d'essais) }\end{array}$} & \multicolumn{2}{|c|}{$\begin{array}{c}\mathrm{N} 205 \\
\text { (sur tour parallèle) }\end{array}$} \\
\hline & 1 & 2 & 3 & 4 & 5 & 6 & 7 & 1 & 2 \\
\hline Type du défaut & $\begin{array}{c}\text { Bague } \\
\text { ext. }\end{array}$ & $\begin{array}{c}\text { Bague } \\
\text { int. }\end{array}$ & $\begin{array}{c}\text { Bague } \\
\text { ext. }\end{array}$ & $\begin{array}{c}\text { Bague } \\
\text { int. }\end{array}$ & $\begin{array}{l}\text { Un sur } \\
\text { chaque } \\
\text { bague }\end{array}$ & $\begin{array}{c}\text { Deux sur } \\
\text { la bague } \\
\text { ext. }\end{array}$ & Bille & \multicolumn{2}{|c|}{ Bague ext. } \\
\hline Gravité du défaut & Petit & Petit & Grand & Grand & Mixte & Mixte & Grand & Petit & Grand \\
\hline $\begin{array}{c}\text { Fréquences } \\
\text { d'échantillonnage } \\
{[\mathrm{Hz}]}\end{array}$ & \multicolumn{7}{|c|}{$65536,32768,16384,4096,1024$} & \multicolumn{2}{|c|}{$\begin{array}{c}65536,32768 \\
16384,1024 \\
\end{array}$} \\
\hline Vitesses de rotation & \multicolumn{7}{|c|}{$50,30,15,10[\mathrm{~Hz}]$} & \multicolumn{2}{|c|}{710 et 2000 tr.min ${ }^{-1}$} \\
\hline
\end{tabular}
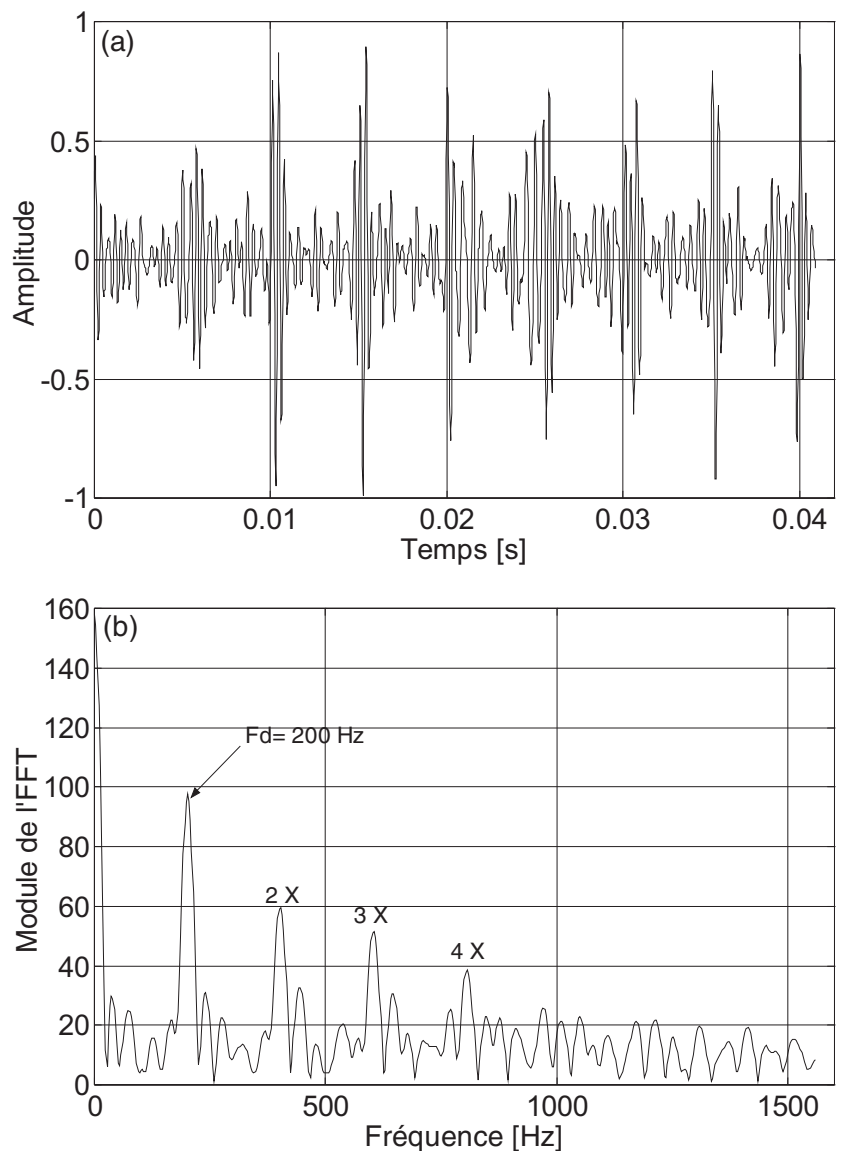

Fig. 6. (a) Signal reconstruit $[1562,5 \mathrm{~Hz}-3125 \mathrm{~Hz}]$, (b) spectre d'enveloppe des coefficients d'ondelettes.

d'un accéléromètre B\&K 4384 avec différentes fréquences d'échantillonnage et pour plusieurs vitesses de rotation. Le tableau 1 regroupe les détails concernant les conditions des expériences.

La figure 8a représente le signal d'accélération d'un roulement du type 6200 sur lequel un défaut a été provoqué sur sa bague extérieure, il est mesuré avec une fréquence d'échantillonnage de 16384 Hz (2048 échantillons). Le roulement tourne à $50 \mathrm{~Hz}$ ce qui donne une fréquence caractéristique d'un défaut sur la bague extérieure (BPFO) égale à environ $131 \mathrm{~Hz}$. Après l'application de la méthode proposée, le signal reconstruit

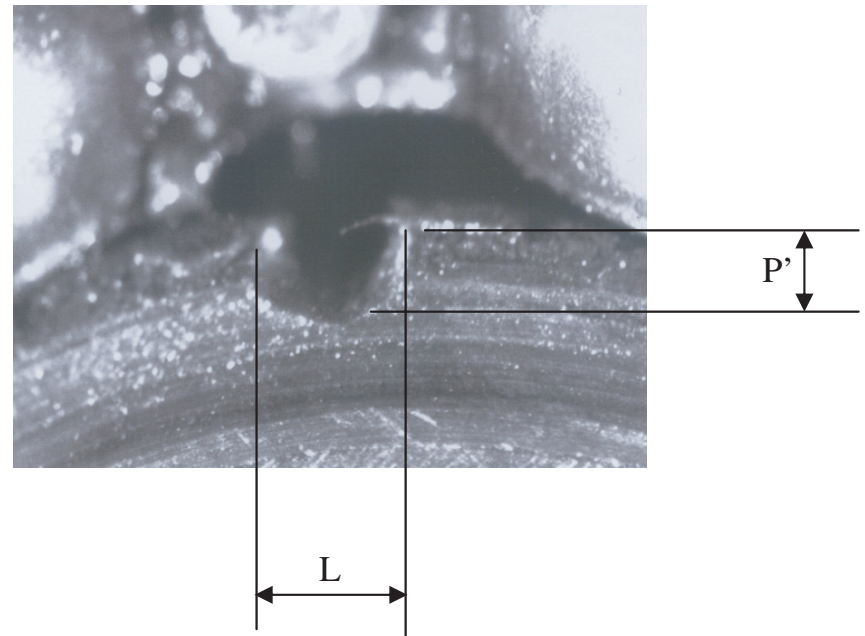

Fig. 7. Forme et dimensions des défauts simulés. Échelle 16/1.

montre clairement les impacts dus au défaut (Fig. 8b), la période des chocs est égale à environ 0,0076 s ce qui correspond parfaitement à la fréquence caractéristique d'un défaut sur la bague extérieure soit $131 \mathrm{~Hz}$. Dans ce cas, le kurtosis étant plus sensible, augmente dans des proportions assez grandes (de 4,31 à 7,18) par rapport au facteur de crête (de 4,64 à 5,23).

Le deuxième cas est celui d'un roulement à rouleaux cylindriques sur lequel un défaut a été provoqué sur sa bague extérieure, le roulement est monté sur un tour parallèle et tourne à 710 tr.min ${ }^{-1}$, dans ce cas la fréquence caractéristique du défaut est égale à environ $47 \mathrm{~Hz}$. Les figures 9a et b représentent respectivement le signal mesuré avec une fréquence d'échantillonnage de $16384 \mathrm{~Hz}$ et celui reconstruit sur lequel apparaissent des impacts dont la période correspond à la fréquence du défaut $(47 \mathrm{~Hz})$. Le kurtosis passe de 6,29 à 10,73 et le facteur de crête de 5,76 à 5,91. Il apparaît que malgré la valeur importante du kurtosis du signal reconstruit, le facteur de crête n'est même pas capable de détecter la présence du défaut.

\subsection{Influence de la gravité du défaut sur la sensibilité des indicateurs scalaires}

Il est évident que les indicateurs scalaires augmentent sensiblement avec la gravité du défaut. En effet pour un 

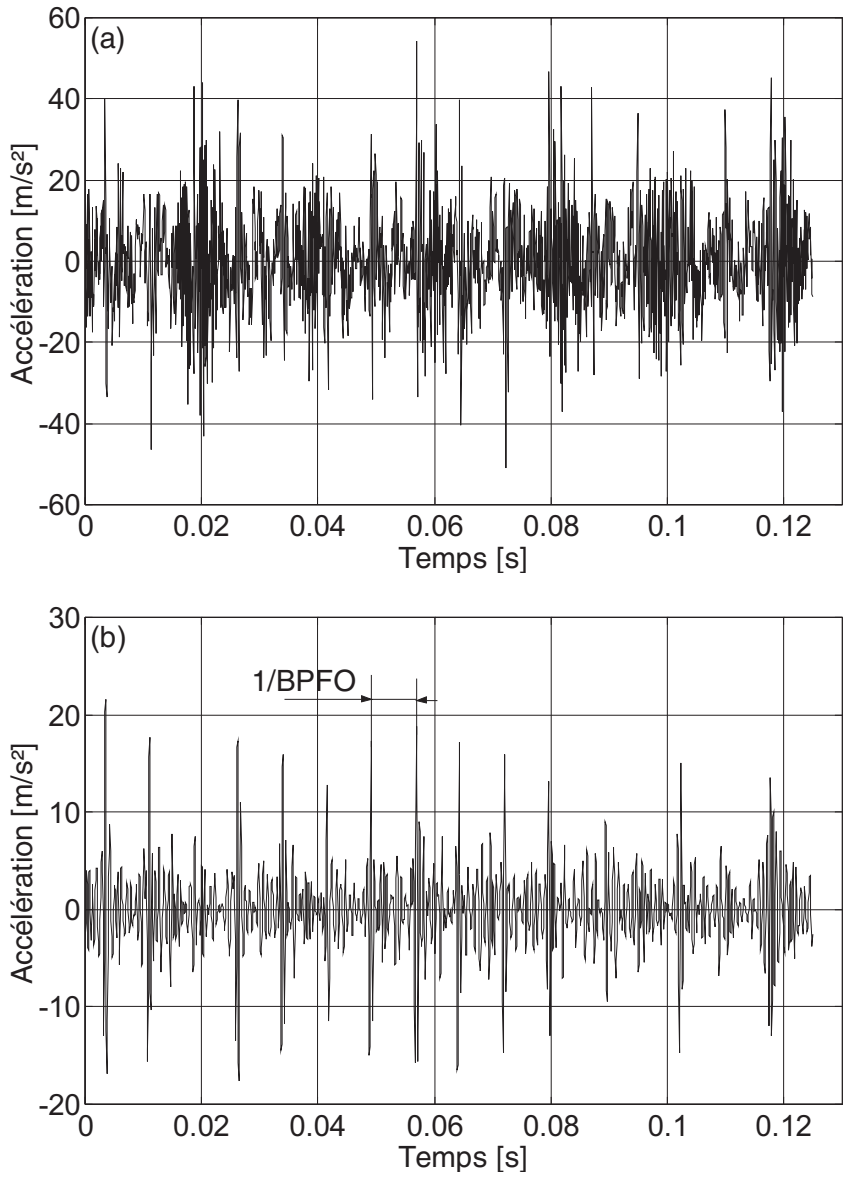

Fig. 8. (a) Signal mesuré, (b) signal reconstruit du roulement avec un défaut sur la bague extérieure; $F_{\mathrm{e}}=16384 \mathrm{~Hz}, N_{\mathrm{r}}=$ $50 \mathrm{~Hz}, N=2048$ échantillons.

roulement en bon état le kurtosis est égal à 3 , si un défaut apparaît, il augmente considérablement en fonction de sa gravité. La figure 10a montre que le facteur de crête des signaux mesurés n'est pas capable de détecter la présence du défaut (même grand). Après l'application de la méthode proposée, le défaut est détecté, même petit, grâce au facteur de crête du signal reconstruit qui dépasse largement 6. Le kurtosis augmente considérablement d'autant que le défaut est grand, en effet plus la dégradation du roulement augmente plus la variation du kurtosis est significative, celle-ci est égale à 1,37 pour le cas d'un petit défaut et 2,78 dans le cas du grand ce qui illustre sa grande sensibilité à la gravité du défaut (Fig. 10b).

\subsection{Influence de la fréquence d'échantillonnage et de la vitesse de rotation}

Les figures 11a et b représentent les résultats d'un défaut sur la bague extérieure, ils montrent que le défaut reste indétectable dans les basses fréquences d'échantillonnage où les valeurs des indicateurs scalaires des signaux mesurés n'atteignent pas les seuils significatifs d'une dégradation du roulement, à savoir trois pour le
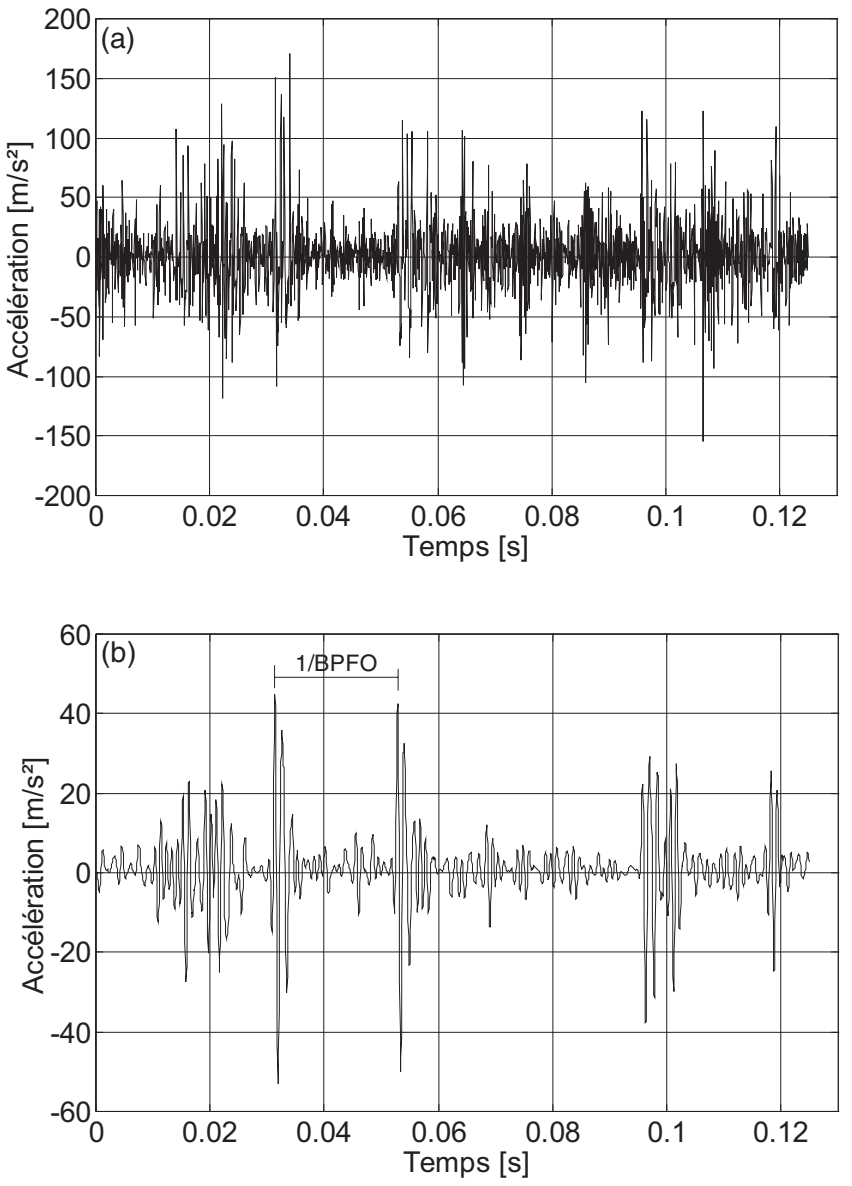

Fig. 9. (a) Signal mesuré, (b) signal reconstruit du roulement avec un défaut sur la bague extérieure; $F_{\mathrm{e}}=16384 \mathrm{~Hz}, N_{\mathrm{r}}=$ 710 tr. $\min ^{-1}, N=2048$ échantillons.

kurtosis et six pour le facteur de crête. Ceux des signaux reconstruits permettent la détection dans trois fréquences d'échantillonnage pour le kurtosis contre une seule pour le facteur de crête. Bien que les deux indicateurs scalaires trouvent leur sensibilité maximale aux hautes fréquences d'échantillonnage, le kurtosis demeure plus fiable pour une prédiction.

Contrairement à la fréquence d'échantillonnage, les figures $12 \mathrm{a}$ et $\mathrm{b}$ montrent que les deux indicateurs scalaires augmentent avec la réduction de la vitesse de rotation, surtout pour les signaux reconstruits, et atteignent leurs valeurs maximales dans la petite vitesse $(10 \mathrm{~Hz})$. Le défaut est détecté par le kurtosis du signal reconstruit dans trois vitesses de rotation contre une seule pour le facteur de crête. En effet une petite vitesse de rotation permet d'avoir une fréquence de choc réduite et par conséquent une fréquence de répétition de choc élevée, ce qui revient aux conditions présentées dans la section 3.2.3.

\subsection{Influence du type du défaut}

La figure 13 montre les kurtosis des signaux reconstruits pour trois défauts : sur la bague externe, sur la 

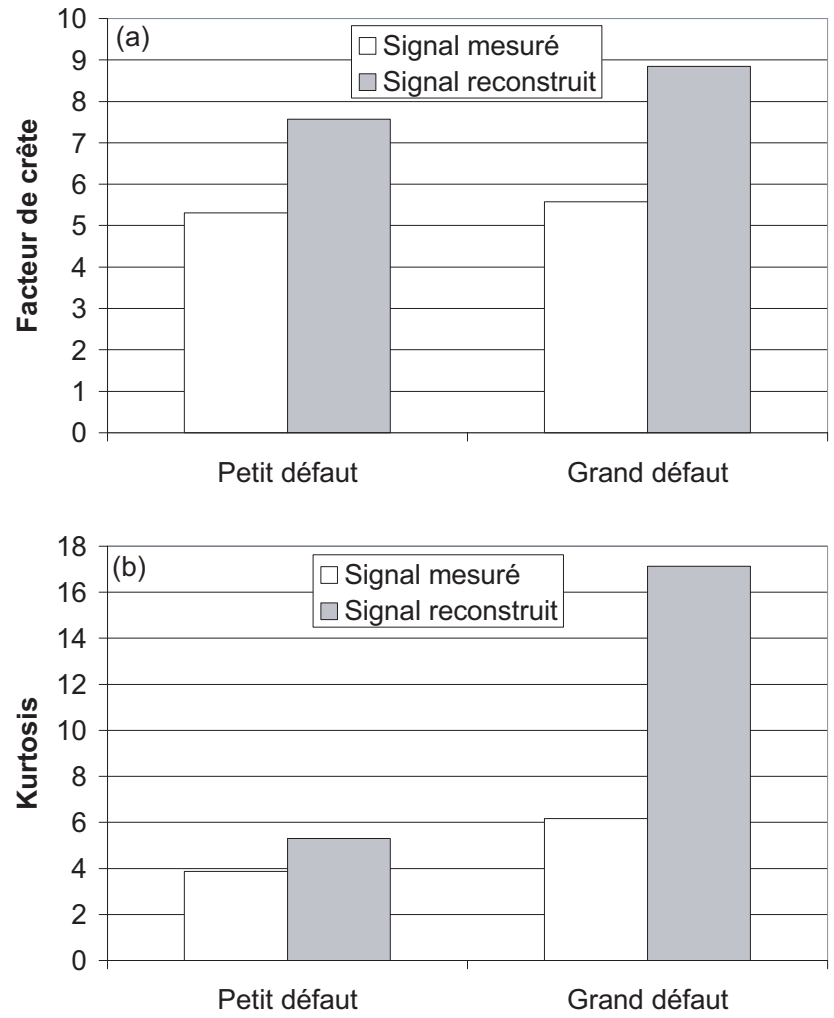

Fig. 10. (a) Facteur de crête et (b) kurtosis du signal mesuré et celui reconstruit dans le cas d'un petit et grand défaut. $F_{\text {e }}=16384 \mathrm{~Hz}$.
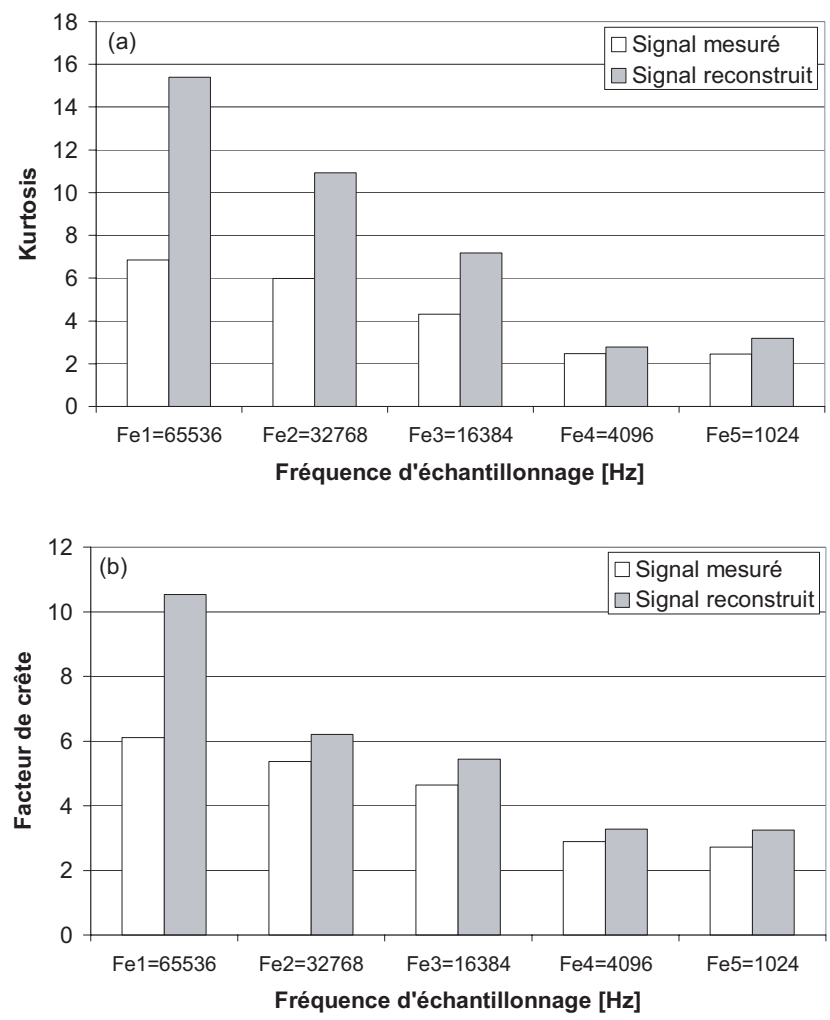

Fig. 11. (a) Kurtosis et (b) facteur de crête des signaux mesurés et ceux reconstruits en fonction de la fréquence d'échantillonnage. $N_{\mathrm{r}}=50 \mathrm{~Hz}$.
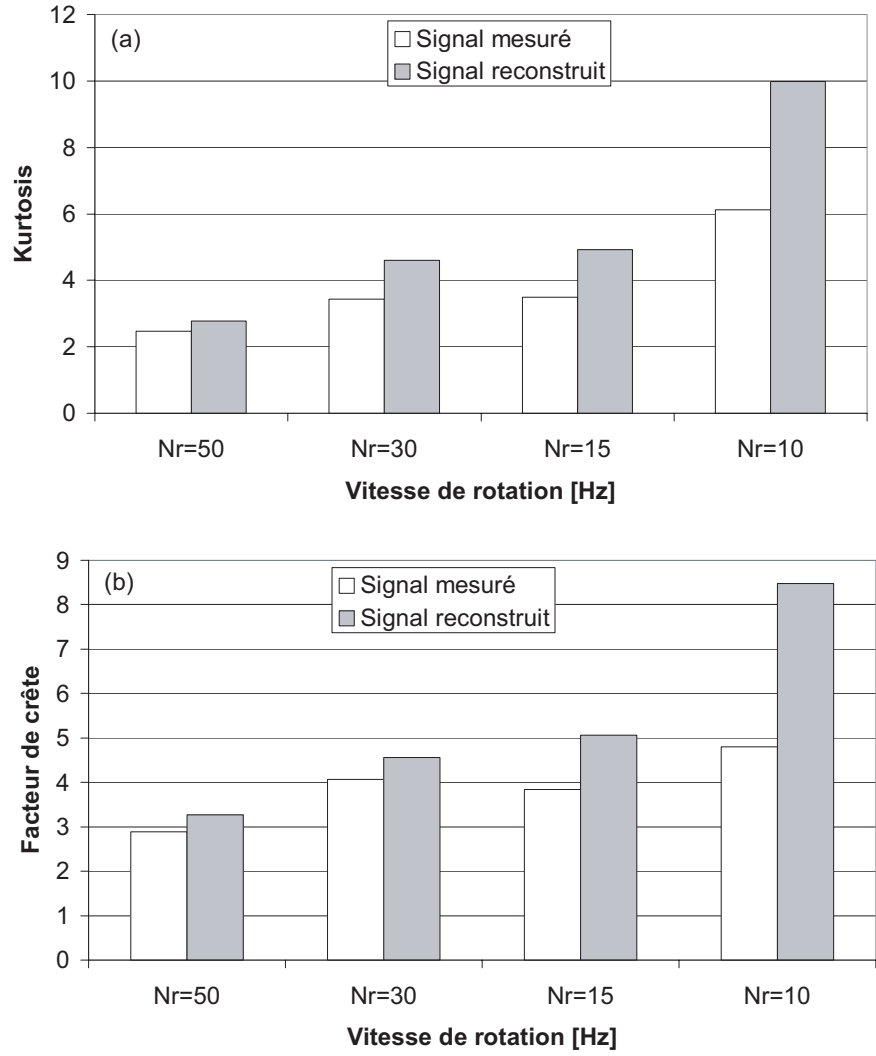

Fig. 12. (a) Kurtosis et (b) facteur de crête des signaux mesurés et ceux reconstruits en fonction de la vitesse de rotation. $F_{\mathrm{e}}=4096 \mathrm{~Hz}$.

bague interne et sur la bille, provoqués avec la même gravité. Les valeurs correspondant au défaut de bille sont les plus significatives surtout dans les plus hautes fréquences d'échantillonnage, conclusion confirmée dans [3]. Pour les autres, celles du défaut sur la bague externe sont prépondérantes. Néanmoins, pour la plus basse fréquence d'échantillonnage, le kurtosis des trois types de défaut est presque identique, ce qui illustre l'intérêt des mesures hautes fréquences.

En conclusion, le kurtosis est plus fiable que le facteur de crête comme indicateur. Des statistiques réalisées sur l'ensemble des 7 roulements (après traitement de 140 signaux) montrent que le kurtosis des signaux reconstruits permet une détection dans $97 \%$ des cas contre $65 \%$ pour le facteur de crête ${ }^{1}$ (Fig. 14).

\section{Application en milieu industriel}

Cette application a été réalisée dans un complexe de raffinage de sucre sur un groupe turbo-alternateur constitué d'une turbine $(\mathrm{T})$ tournant à $6000 \mathrm{tr}^{-\mathrm{min}^{-1}}$ $(100 \mathrm{~Hz})$, un réducteur $(\mathrm{R})$ dont le rapport est (6000/1500) et un alternateur (A) dont la vitesse est de 1500 tr. $\min ^{-1}(25 \mathrm{~Hz})$. Différentes mesures globales de la

${ }^{1}$ Les seuils de détection retenus sont trois pour le kurtosis et six pour le facteur de crête. 


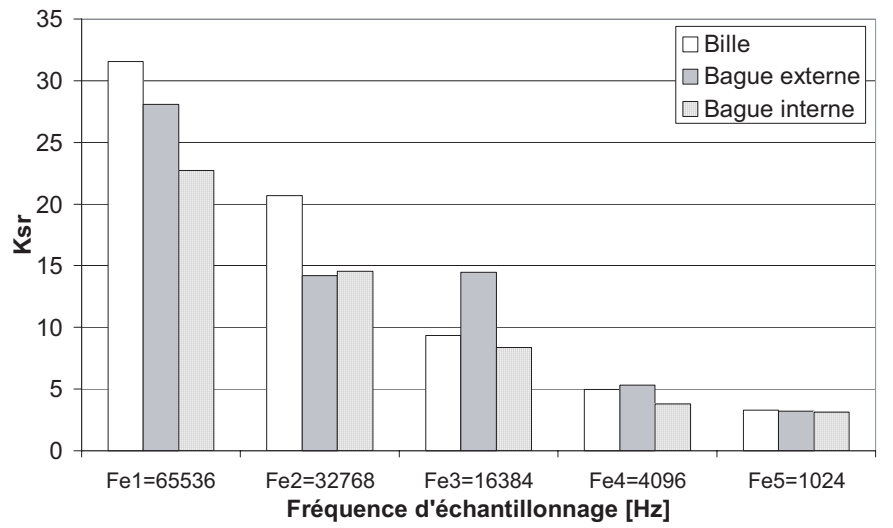

Fig. 13. Kurtosis des signaux reconstruits en fonction de la fréquence d'échantillonnage pour trois types de défauts. $N_{\mathrm{r}}=$ $30 \mathrm{~Hz}$.

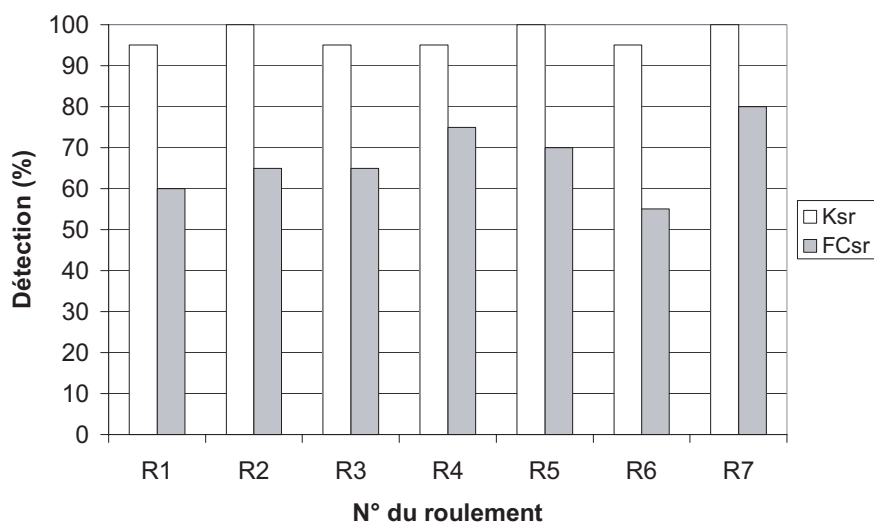

Fig. 14. Taux de détection du kurtosis et du facteur de crête des signaux reconstruits dans toutes les configurations pour l'ensemble des roulements.

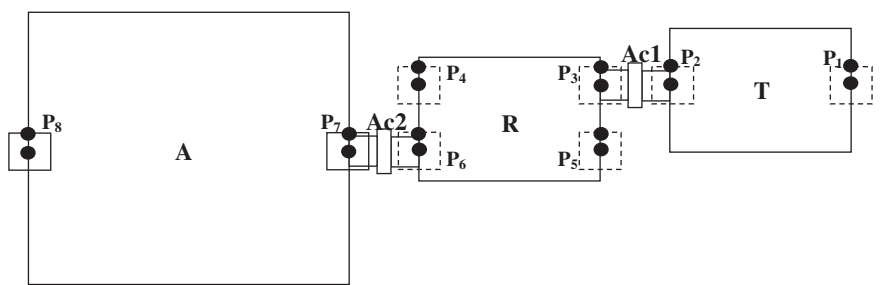

Fig. 15. Schéma simplifié du groupe turbo-alternateur et des points de mesure.

tendance vibratoire faites antérieurement ont laissé penser à une anomalie de fonctionnement du groupe, une vitesse efficace maximale de $8 \mathrm{~mm} . \mathrm{s}^{-1}$ a été enregistrée au point 2 , ce qui représente une valeur classée « médiocre » pour ce type de machine selon ISO 2372. Plusieurs nouvelles mesures ont été réalisées sur huit points dans les deux directions (horizontale et verticale). La figure 15 représente le schéma du groupe et des points de mesures.

Après l'application de la méthode proposée, il apparaît que le kurtosis du signal reconstruit du point 2 dépasse trois $(3,66)$, voire très inquiétant pour le point 8 où il atteint 9,47 (voir Tab. 2). Le spectre du point 2 montre que la fréquence des chocs est égale à $50 \mathrm{~Hz}$
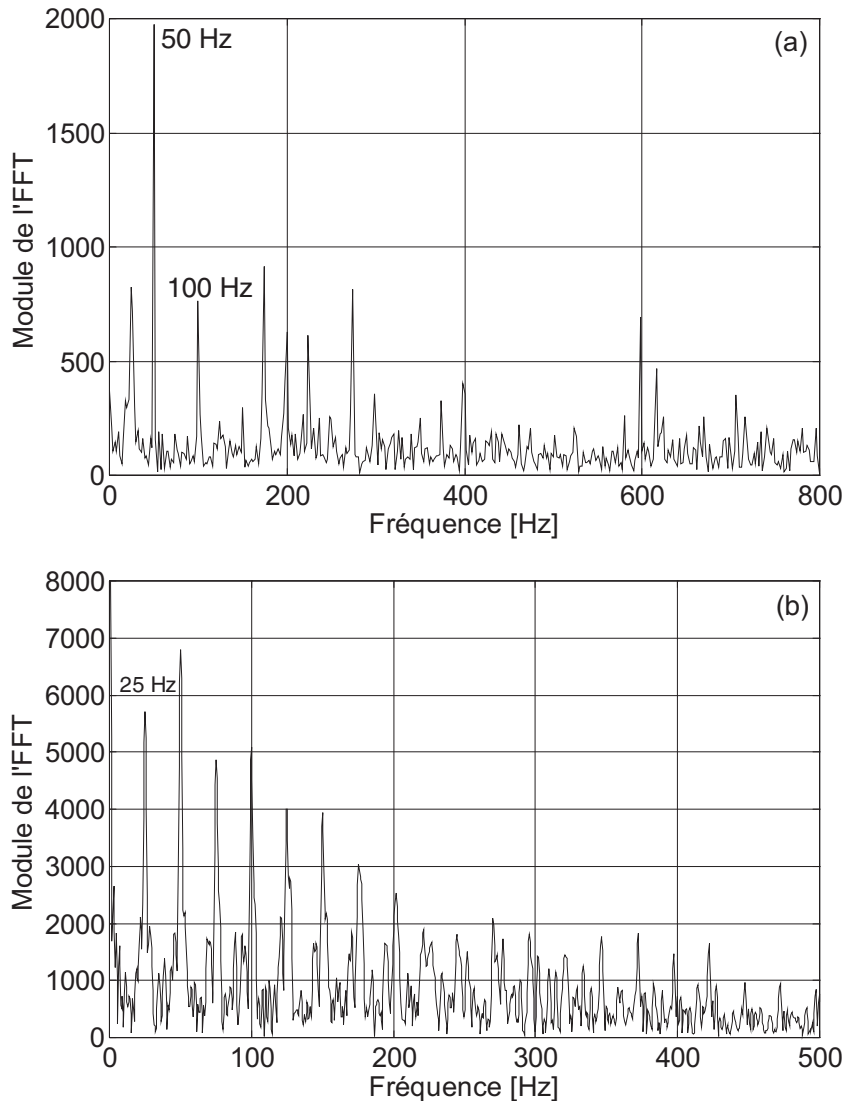

Fig. 16. (a) Spectre au point 2, (b) spectre d'enveloppe des coefficients d'ondelettes au point 8 .

(Fig. 16a) ce qui correspond parfaitement à une fréquence d'instabilité du palier ${ }^{2}$. Dans celui des coefficients d'ondelettes du signal reconstruit du point 8, la fréquence des chocs ne correspond à aucune fréquence caractéristique de défaut mais à celle de la fréquence de rotation du roulement $(25 \mathrm{~Hz})$ ainsi que plusieurs de ces harmoniques (Fig. 16b), ce qui laisse penser que le jeu de montage est trop grand (le roulement tourne dans l'arbre). Bien évidemment des consignes ont été données à cet effet.

\section{Conclusion}

Dans cet article l'analyse multirésolution en ondelettes est proposée comme un outil efficace permettant d'améliorer la sensibilité des indicateurs scalaires pour la détection des défauts induisant des forces impulsives périodiques. Adaptée à cette fin, plusieurs de ces paramètres d'analyse ont été optimisés, de plus elle offre une visualisation fréquentielle claire du défaut et permet une détection fiable aussi bien pour les hautes fréquences que pour les plus basses d'entre elles. Les résultats des mesures expérimentales réalisées sur des roulements défectueux montrent la validité de cette méthode pour des hautes

\footnotetext{
${ }^{2}$ Il a été montré qu'un palier instable génère des chocs lors-
} qu'il y a présence de fouettement d'huile. 
Tableau 2. Résultats du turbo-alternateur.

\begin{tabular}{|c|c|c|c|}
\hline \multicolumn{2}{|c|}{ Point de mesure } & 2 & 8 \\
\hline & Type & Palier lisse & $\begin{array}{c}\text { Roulement du type SKF NU } \\
330 \text { MIC } 3\end{array}$ \\
\hline \multirow{2}{*}{ kurtosis } & Signal mesuré & 3,11 & 4,96 \\
\hline & Signal reconstruit & 3,66 & 9,47 \\
\hline \multirow{2}{*}{$\begin{array}{l}\text { Facteur de } \\
\text { crête }\end{array}$} & Signal mesuré & 3,67 & 2,11 \\
\hline & Signal reconstruit & 4,39 & 6,66 \\
\hline
\end{tabular}

fréquences d'échantillonnage et de faibles vitesses de rotation, la grande sensibilité du kurtosis à ces deux paramètres ainsi qu'au type et à la gravité du défaut a été démontrée. Le kurtosis semble plus fiable que le facteur de crête et demeure ainsi un indicateur précieux. Il a permis, sur 140 signaux traités dans différentes configurations, une détection dans $97 \%$ des cas, ce qui le place dans l'avant garde des indicateurs fiables de l'état de dégradation des roulements. L'application de la méthode proposée en milieu industriel confirme sa validité ainsi que son éventuelle extension aux autres défauts induisant des chocs tels que les engrenages, objet de notre prochaine étude.

\section{Références}

[1] A. Boulenger, C. Pachaud, Diagnostic vibratoire en maintenance préventive, Dunod, 1998

[2] C. Pachaud, R. Salvetat, C. Fray, Crest factor and kurtosis contributions to identify defects inducing periodical impulsive forces, Mech. Syst. Signal Processing 11 (1997) 903-916

[3] R.B.W. Heng, M.J. Nor, Statistical analysis of sound and vibration signals for monitoring rolling element bearing condition, Applied Acoustics 53 (1998) 211-226

[4] J.P. Dron, F. Bolaers, L. Rasolofondraibe, Optimisation de la détection des défauts de roulements par débruitage des signaux par soustraction spectrale, Mécanique \& Industries 4 (2003) 213-219

[5] J.P. Dron, F. Bolaers, L. Rasolofondraibe, Improvement of the sensitivity of the scalar indicators (crest factor and kurtosis) using a de-noising method by spectral subtraction: application to the detection of defects in ball bearings, J. Sound Vib. 270 (2004) 61-73

[6] G.K. Chaturvedi, D.W. Thomas, Bearings faults detection using adaptive noise cancelling, J. Mechanical Design 104 (1982) 280-289

[7] Y. Shao, K. Nezu, Design of mixture de-noising for detecting faulty bearing signals, J. Sound Vibration 282 (2005) 899-917

[8] D.L. Donoho, De-noising by soft thresholding, IEEE Trans. Information Theory 41 (1995) 613-627

[9] L. Jing, Feature extraction based on Morlet wavelet and its application for mechanical fault diagnosis, J. Sound Vib. 234 (2000) 35-148

[10] W. Peter, Wavelets analysis-A flexible and efficient fault diagnostic method for rolling element bearing, 7th International Congress on Sound and Vibration, Germany, 4-7 July 2000, 507-514

[11] Q. Sun, Y. Tang, Singularity analysis using continuous wavelet transform for bearing fault diagnosis, Mechanical Systems and Signal Processing 16 (2002) 1025-1041

[12] W.J. Wang, P.D. MacFadden, Application of wavelets to gearbox vibration signals for fault detection, J. Sound Vib. 192 (1996) 927-939

[13] H. Zheng, Z. Li, X. Chen, Gear faults diagnosis based on continuous wavelet transform, Mechanical Systems and Signal Processing 16 (2002) 447-457

[14] A. Yoshida, Y. Ohue, H. Ishikawa, Diagnosis of tooth surface failure by wavelet transform of dynamic characteristics, Tribology Int. 33 (2000) 273-279

[15] R. Rubini, U. Meneghetti, Application of the envelope and wavelet transform analyses for the diagnosis of incipient faults in ball bearings, Mechanical Systems and Signal Processing 15 (2001) 287-302

[16] S. Brabhakar, A.R. Mohanty, A.S. Sekhar, Application of discrete wavelet transform for detection of ball bearings race faults, Tribology Int. 35 (2002) 793-800

[17] A. Djebala, N. Ouelaa, N. Hamzaoui, S. Guenfoud, Détection précoce des défauts de roulements par analyse multirésolution en ondelettes, First International Congress Design and Modeling of Mechanical Systems, Tunisia 23-25 March 2005

[18] K. Chinmaya, A.R. Mohanty, Monitoring gear vibrations through motor current signature analysis and wavelet transform, Mechanical Systems and Signal Processing 20 (2006) 158-187

[19] C.K. Sung, H.M. Tai, C.W. Chen, Locating defects of gear system by the technique of wavelet transform, Mechanism Machine Theory 35 (2000) 1169-1182

[20] N.G. Nikolaou, I.A. Antoniadis, Rolling element bearing fault diagnosis using wavelet packets, NDT \& E Int. 35 (2002) 197-205

[21] E. Ericsson, N. Grip, E. Johansson, L.-E. Persson, R. Sjöberg, J.O. Strönberg, Towards automatic detection of local bearing defects in rotating machines, Mechanical Systems and Signal Processing 19 (2005) 509-535

[22] M. Boltezar, I. Simonovski, M. Furlan, Faults detection in DC electro motors using the continuous wavelet transform, Meccanica 38 (2003) 251-264

[23] D.M. Yang, A.F. Stronach, P. MacConnell, The application of advanced signal processing techniques to induction motor bearing condition diagnosis, Meccanica 38 (2003) 297-308 